\title{
Methods Supplement
}

\section{Sample Locations}

The study sections were either collected by the authors $(1-2,10,13-14)$ or provided by colleagues. We wish to thank in particular Brooks Ellwood for sections 3-4, Leo Krystyn for section 5, Michael Brookfield for section 6, Micha Horacek for sections 7-9, Steve Grasby for section 11, Paul Wignall for section 12, and Hiroyoshi Sano for sections 15-16 (see Tables DR1DR3 for section identification). The sections have a near-global distribution: 1-4 are from the South China craton, 5-9 from non-Chinese Tethyan locales, 10-11 and 12-14 from the northern and western margins of Pangea, respectively, and 15-16 from the Panthalassic region. The sections are varied in terms of tectonic setting: 1 and 7-14 represent stable continent margins, 2-4 represent carbonate platforms, 5-6 represent rift blocks (unstable continent margins), and 15-16 represent deep-marine settings. The dominant lithologies of the sections are also varied: Tethyan locales are characterized by carbonates (2-4, 7-9), marls (1), or mixed lithologies (5-6), nonTethyan continent-margin locales are mainly silty shales (10-14), and the deep-marine sections consist of radiolarian cherts (15-16; Table DR3).

Time scale

Calculations of sedimentary rates require adequate age control. The Phanerozoic time scale of Gradstein et al. (2004) gives dates of 253.8, 251.0, 249.7, and 245.0 Ma for the Wuchiapingian/Changhsingian, Changhsingian/Induan (Permian/Triassic), Induan/Olenekian, and Olenekian/Anisian (Early/Middle Triassic) stage boundaries, respectively (Fig. DR1). However the Late Permian-Early Triassic time scale has been under revision in recent years based on high-resolution radiometric dates for volcanic ash layers in Wuchiapingian (early Upper Permian) through Anisian (Middle Triassic) units (Mundil et al., 2004; Lehrmann et al., 2006; Ovtcharova et al., 2006; Galfetti et al., 2007). These studies have all used the CA-TIMS ("chemical abrasion thermal ionization mass spectrometry") method of $\mathrm{U}-\mathrm{Pb}$ dating of single zircons, which yields ages with an analytical uncertainty typically of $+/-0.2$ to $0.3 \mathrm{~m} . \mathrm{y}$. (Mattinson, 2005). This method generally avoids artifacts associated with secondary $\mathrm{Pb}$ loss but cannot resolve all uncertainties related to inheritance. These studies have revised the ages of the various stratigraphic boundaries considered in this study, yielding ages that are $\sim 1$ to 2 m.y. older 


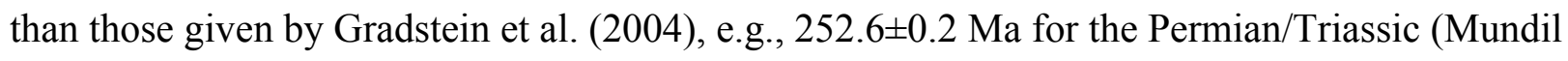

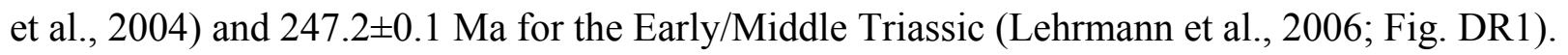

The radiometric dating studies above provide constraints on the durations of the two main time-stratigraphic units of interest to this study, the Changhsingian stage of the Late Permian and the Griesbachian substage of the Early Triassic. While the Changhsingian/Griesbachian (i.e., Permian/Triassic) boundary has been securely dated at 252.6 $\pm 0.2 \mathrm{Ma}$ (Mundil et al., 2004), the ages of the base of the Changhsingian and the top of the Griesbachian remain somewhat uncertain (Fig. DR1). Mundil et al. (2004) obtained a date of 257.3 $\pm 0.3 \mathrm{Ma}$ for an ash layer of late Wuchiapingian age, from which the age of the base of the Changhsingian can be interpolated as $\sim 256.0 \pm 1.0 \mathrm{Ma}$, making the duration of the Changhsingian stage $3.4 \pm 1.2 \mathrm{~m}$.y. Galfetti et al. (2007) obtained a date of 251.22 $\pm 0.20 \mathrm{Ma}$ for an ash layer of early Smithian age, from which the top of the Induan stage can be interpolated as $251.3 \pm 0.2 \mathrm{Ma}$, making the duration of the Induan Stage $1.3 \pm 0.4$ m.y. In a recent review paper, Mundil et al. (2010) proposed an even shorter duration for the Induan ( 1.0 m.y.) based on ages for the Permian/Triassic and Induan/Olenekian boundaries of $252.3 \pm 0.3 \mathrm{Ma}$ and $251.3 \pm 0.2 \mathrm{Ma}$, respectively. The Griesbachian/Dienerian boundary has not been radiometrically dated yet, so estimates of the durations of the two Induan substages can be derived only from their relative stratigraphic thicknesses. The 0.7 and 0.6 m.y. durations assigned the Griesbachian and Dienerian, respectively, by Gradstein et al. (2004) remain consistent with recent radiometric dating results, and a duration of $0.7 \pm 0.4$ m.y. for the Griesbachian was used in the rate calculations of the present study.

\section{Sedimentation rate and flux calculations}

Linear sedimentation rates (LSR) were calculated for a time-stratigraphic unit of interest (i.e., the Changhsingian or Griesbachian) based on its thickness at a given study site and its estimated duration based on recent radiometric dating studies:

$$
\operatorname{LSR}=\varphi / \tau
$$

where $\varphi$ is thickness in units of meters $(\mathrm{m})$, and $\tau$ is duration in units of millions of years (m.y.). Where the full thickness of the Changhsingian or Griesbachian was not sampled in this study, additional published sources for the same region were consulted in order to arrive at a reliable total thickness estimate for purposes of LSR calculations. For a limited number of study sections, LSRs were determined on the basis of results of spectral analyses (e.g., Algeo et al., 2010a, 2010b). 
Note that modern sedimentation rates (as in Figure 4) cannot be compared directly with ancient rates (Table DR1), which are invariably lower by an order-of-magnitude or more owing to timeaveraging effects (Wilkinson et al., 1991). Bulk accumulation rates (BAR) were calculated as:

$$
\mathrm{BAR}=\operatorname{LSR} \times \rho
$$

where BAR is in units of $\mathrm{g} \mathrm{m}^{-2} \mathrm{yr}^{-1}$, LSR is in units of $\mathrm{m} \mathrm{m.y.} .^{-1}$, and $\rho$ is bulk sediment density in units of $\mathrm{g} \mathrm{cm}^{-3}$. Owing to lack of bulk sediment density data, a value of $2.5 \mathrm{~g} \mathrm{~cm}^{-3}$ was used in all BAR calculations. Uncertainties in BARs resulting from this assumption are small: most organic-poor siliciclastic and carbonate sedimentary rocks have bulk densities within $10 \%$ of this value (Schmoker, 1977). The fluxes of individual sediment components were calculated as:

$$
\mathrm{f}_{\mathrm{A}}=\mathrm{BAR} \times \mathrm{C}_{\mathrm{A}}
$$

where $\mathrm{fA}$ is the flux of component $\mathrm{A}$ (i.e., $\mathrm{CaCO}_{3}$, quartz, or clays) in units of $\mathrm{g} \mathrm{m}^{-2} \mathrm{yr}^{-1}$, and $\mathrm{C}_{\mathrm{A}}$ is the concentration of component $\mathrm{A}$ in percent (as determined by equations 4-6).

\section{Calculation of lithologic fractions}

Lithologic compositions were calculated based on elemental concentration data. Total inorganic carbon (TIC) was determined by difference from measurements of total carbon and TOC using an Eltra $2000 \mathrm{C}-\mathrm{S}$ analyzer and major oxide concentrations were determined using a Rigaku 3040 X-ray fluorescence (XRF) spectrometer, both located at the University of Cincinnati. Percentages of the three dominant components of the study sections were calculated as follows (cf. Algeo et al., 2007):

$$
\begin{aligned}
& \mathrm{CaCO}_{3}(\%)=\mathrm{TIC}_{\text {meas }} \times 100 / 12.0 \\
& \text { Clay minerals }(\%)=\mathrm{Al}_{\text {meas }} \times 100 / \kappa_{1} \\
& \text { Quartz }(\%)=\mathrm{SiO}_{2 \text { (meas) }}-\left(\mathrm{Al}_{\text {meas }} / 27.0 \times \kappa_{2} \times 60.1\right)
\end{aligned}
$$

Equation 4 is a standard formula for calculating the amount of calcium carbonate in a sample from the measured concentration of total inorganic carbon (TIC). Equations 5 and 6 are formulas for calculating model amounts of clay minerals and quartz in a sample based on measured $\mathrm{Al}$ and $\mathrm{SiO}_{2}$ concentrations. Note that "quartz" includes both detrital quartz and biogenic silica (i.e., chert). The coefficients 12.0, 27.0, and 60.1 represent the molar weights in grams of C, $\mathrm{Al}$, and $\mathrm{SiO}_{2}$, respectively. The constants $\kappa_{1}$ and $\kappa_{2}$ represent the average concentration of $\mathrm{Al}$ and the average 
molar Si:Al ratio of the clay mineral assemblage, respectively, and values for these constants in each formation were selected to minimize variance about a mean sum of $100 \%$ for the samples as a group. The actual values chosen for $\kappa_{1}$ ranged from 1.50 to 2.50 (mean 2.00 \pm 0.33 ) and those for $\kappa_{2}$ ranged from 11.1 to 16.0 (mean $12.9 \pm 1.3$ ). These values are consistent with the dominant clay mineral in most of the study sections being illite, which typically contains $13-15 \% \mathrm{Al}$ and has a molar Si:Al ratio of 2.0 (dictated by its TOT structure; Grim, 1968). Other clay minerals are characterized by average $\mathrm{Al}$ concentrations and molar Si:Al ratios that differ from these values, e.g., chlorite typically contains 9-13\% Al and has a molar $\mathrm{Si}$ :Al ratio of 1.0-2.0 (depending on the degree of substitution for Al in its octahedral layers; Grim, 1968).

\section{References}

Algeo, T.J., Hannigan, R., Rowe, H., Brookfield, M., Baud, A., Krystyn, L., and Ellwood, B.B., 2007, Sequencing events across the Permian-Triassic boundary, Guryul Ravine (Kashmir, India): Palaeogeography, Palaeoclimatology, Palaeoecology, v. 252, p. 328-346, doi:10.1016/j.palaeo.2006.11.050.

Galfetti, T., Bucher, H., Ovtcharova, M., Schaltegger, U., Brayard, A., Brühwiler, T., Goudemand, N., Weissert, H., Hochuli, P.A., Cordey, F., and Guodun, K., 2007, Timing of the Early Triassic carbon cycle perturbations inferred from new $\mathrm{U}-\mathrm{Pb}$ ages and ammonoid biochronozones: Earth and Planetary Science Letters, v. 258, p. 593-604.

Gradstein, F., Ogg, J., and Smith, A., 2004, A Geologic Time Scale 2004, Cambridge University Press, Cambridge, 589 pp.

Grim, R.E., 1968, Clay Mineralogy, $2^{\text {nd }}$ ed., McGraw-Hill, New York, 596 pp.

Lehrmann, D.J., Ramezani, J., Bowring, S.A., Martin, M.W., Montgomery, P., Enos, P., Payne, J.L., Orchard, M.J., Wang, H., and Wei, J., 2006, Timing of recovery from the end-Permian extinction: geochronologic and biostratigraphic constraints from south China: Geology, v. 34, p. 1053-1056.

Mattinson, J.M., 2005, Zircon U-Pb chemical abrasion (“CA-TIMS”) method: combined annealing and multistep partial dissolution analysis for improved precision and accuracy of zircon ages: Chemical Geology, v. 220, p. 47-66. 
Mundil, R., Ludwig, K.R., Metcalfe, I., and Renne, P.R., 2004, Age and timing of the Permian mass extinctions: U/Pb dating of closed-system zircons: Science, v. 305, p. 1760-1763.

Mundil, R., Pálfy, J., Renne, P.R., and Brack, P., 2010, The Triassic time scale: New constraints and a review of geochronological data. In: Lucas, S.G. (Ed.), The Triassic Timescale. Geological Society of London, Special Publication 334, pp. 41-60.

Ovtcharova, M., Bucher, H., Schaltegger, U., Galfetti, T., Brayard, A., and Guex, J., 2006, New Early to Middle Triassic U-Pb ages from South China: Calibration with ammonoid biochronozones and implications for the timing of the Triassic biotic recovery: Earth and Planetary Science Letters, v. 243, p. 463-475.

Schmoker, J.W., 1977, The relationship between density and gamma-ray intensity in the Devonian shale sequence, Lincoln County, West Virginia. In: Overbey, W.K., Jr., Hunt, A.E., and Komar, C.A. (Eds.), Proceedings of the First Eastern Gas Shales Symposium, Morgantown Energy Research Center, Morgantown, West Virginia, pp. 266-271.

Wilkinson, B.H., Opdyke, B.N., and Algeo, T.J., 1991, Time partitioning in cratonic carbonates: Geology, v. 19, p. 1093-1096, doi:10.1130/0091-7613(1991)019<1093:TPICCR>2.3.CO;2. 
Gradstein et al. (2004)

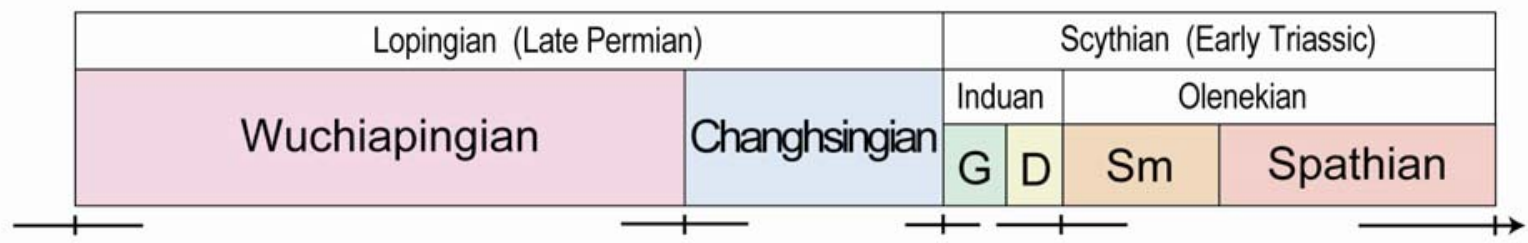

\section{Revised timescale}

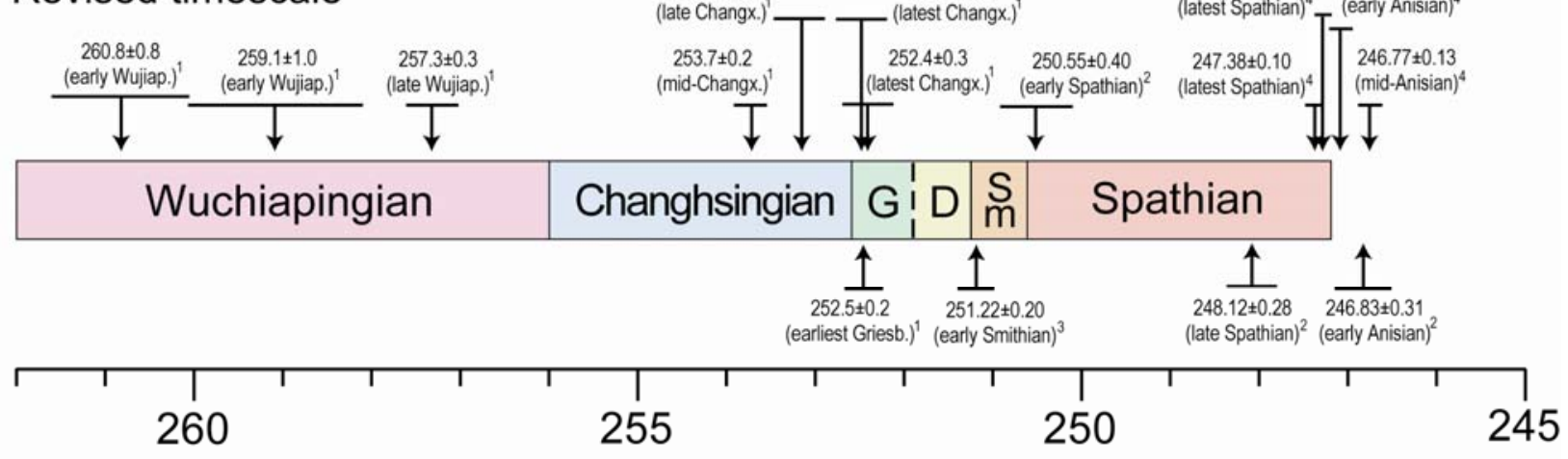

Age (Ma)

Figure DR1. Late Permian-Early Triassic timescale of Gradstein et al. (2004; above) and revised timescale based on recent $\mathrm{U}-\mathrm{Pb}$ radiometric dating studies (below). Sources: $1=$ Mundil et al. (2004), 2 = Ovtcharova et al. (2006), 3 = Galfetti et al. (2007), and 4 = Lehrmann et al. (2006). Abbreviations: $\mathrm{G}=$ Griesbachian, $\mathrm{D}=$ Dienerian, and $\mathrm{Sm}=$ Smithian. Age uncertainties for stratigraphic boundaries and radiometric dates are shown by horizontal lines. 
Table DR1. Linear sedimentation rates (LSR) for Changhsingian (uppermost Permian) and Griesbachian (lowermost Triassic) units

\begin{tabular}{|c|c|c|c|c|c|c|c|c|c|}
\hline \multirow[b]{2}{*}{$\begin{array}{l}\text { No } \\
\text { - }\end{array}$} & \multirow[b]{2}{*}{ Section } & \multicolumn{3}{|c|}{ CHANGXINGIAN } & \multicolumn{3}{|c|}{ GRIESBACHIAN } & \multirow{2}{*}{$\begin{array}{c}\text { Gr/Ch } \\
\text { LSR } \\
\%^{2}\end{array}$} & \multirow[b]{2}{*}{ Data Sources } \\
\hline & & $\begin{array}{c}\text { thickne } \\
\text { ss } \\
\text { m }\end{array}$ & $\begin{array}{c}\text { duratio } \\
\text { n } \\
\text { Myr }\end{array}$ & $\begin{array}{l}\text { LSR }^{1} \\
\mathrm{~m} \mathrm{Myr}^{-1}\end{array}$ & $\begin{array}{c}\text { thicknes } \\
\text { s } \\
m\end{array}$ & $\begin{array}{c}\text { duratio } \\
\text { n } \\
\text { Myr }\end{array}$ & $\begin{array}{l}\text { LSR }^{1} \\
\mathrm{~m} \mathrm{Myr}^{-1}\end{array}$ & & \\
\hline 1 & Chaohu, Anhui & 48.0 & 3.4 & 14.1 & 60.0 & 0.7 & 85.7 & 507 & Li et al., 2007; Zhao et al., 2007 \\
\hline 2 & Dawen, Guizhou & 120.0 & 3.4 & 35.3 & 160.0 & 0.7 & 228.6 & 548 & Lehrmann et al., 2001; Chen et al., 2009 \\
\hline 3 & Nhi Tao, Vietnam & 1.3 & 0.03 & 42.9 & 6.0 & 0.06 & 96.0 & 124 & Algeo et al., 2007a, 2008; Son et al., 2007 \\
\hline 4 & Lung Cam, Vietnam & 2.6 & 0.06 & 42.9 & 2.4 & 0.03 & 96.0 & 124 & Son et al., 2007; Luu et al., 2009 \\
\hline 5 & Spiti, India & 1.0 & 3.4 & 0.3 & 1.0 & 0.7 & 1.4 & 386 & Orchard \& Krystyn, 1998; Krystyn et al., 2007 \\
\hline 6 & Guryul Ravine, India & 20.0 & 3.4 & 5.9 & 8.0 & 0.7 & 11.4 & 94 & Algeo et al., 2007b; Korte et al., 2010 \\
\hline 7 & Zal, Iran & 40.0 & 3.4 & 11.8 & 120.0 & 0.7 & 171.4 & 1357 & $\begin{array}{l}\text { Korte et al., 2004; Kozur, 2007; Horacek et al., } \\
2007 \mathrm{~b}\end{array}$ \\
\hline 8 & Bulla, Italy & 120.0 & 3.4 & 35.3 & 90.0 & 0.7 & 128.6 & 264 & Twitchett \& Wignall, 1996; Cassinis \& Perotti, \\
\hline 9 & Uomo, Italy & 120.0 & 3.4 & 35.3 & 90.0 & 0.7 & 128.6 & 264 & $\begin{array}{l}2007 a \\
200 \text {, }\end{array}$ \\
\hline 10 & Spitsbergen & 40.0 & 3.4 & 11.8 & 60.0 & 0.7 & 85.7 & 629 & R. Twitchett (pers. comm. 2009) \\
\hline 11 & $\begin{array}{l}\text { West Blind Fiord, } \\
\text { Arctic }\end{array}$ & 20.0 & 3.4 & 5.9 & 100.0 & 0.7 & 142.9 & 2329 & $\begin{array}{l}\text { Henderson \& Baud, 1997; Grasby \& } \\
\text { Beauchamp, } 2009\end{array}$ \\
\hline 12 & Ursula Creek & 1.0 & 0.5 & 2.0 & 100.0 & 0.7 & 142.9 & 7043 & $\begin{array}{l}\text { Wignall \& Newton, 2003; Wignall (pers. comm. } \\
\text { 2009) }\end{array}$ \\
\hline 13 & $\begin{array}{l}\text { Crooked Creek, } \\
\text { Alberta }\end{array}$ & 1.0 & 0.5 & 2.0 & 80.0 & 0.7 & 114.3 & 5614 & Hays et al., 2007 \\
\hline 14 & Opal Creek, Alberta & 1.0 & 0.5 & 2.0 & 80.0 & 0.7 & 114.3 & 5614 & Henderson, 1997 \\
\hline 15 & $\begin{array}{l}\text { Gujo-Hachiman, } \\
\text { Japan }\end{array}$ & 7.0 & 1.1 & 6.4 & 0.7 & 0.4 & 1.6 & -74 & Algeo et al., 2010a, 2010b \\
\hline 16 & Ubara, Japan & 0.9 & 0.2 & 4.5 & 0.4 & 0.2 & 2.3 & -48 & Algeo et al., 2010b \\
\hline
\end{tabular}

${ }^{1}$ Calculated per Equation 1 in Methods Section.

${ }^{2}$ Represents the percentage change in LSR from the Changxingian (Ch) to the Griesbachian (Gr). 


\section{References for Table DR1}

Algeo, T.J., Ellwood, B.B., Nguyen, T.K.T., Rowe, H., and Maynard, J.B., 2007a, The Permian-Triassic boundary at Nhi Tao, Vietnam: Evidence for recurrent influx of sulfidic watermasses to a shallow-marine carbonate platform: Palaeogeography, Palaeoclimatology, Palaeoecology, v. 252, p. 304-327.

Algeo, T.J., Hannigan, R., Rowe, H., Brookfield, M., Baud, A., Krystyn, L., and Ellwood, B.B., 2007b, Sequencing events across the Permian-Triassic boundary, Guryul Ravine (Kashmir, India): Palaeogeography, Palaeoclimatology, Palaeoecology, v. 252, p. 328346.

Algeo, T.J., Hinnov, L., Moser, J., Maynard, J.B., Elswick, E., Kuwahara, K., and Sano, H., 2010a, Changes in productivity and redox conditions in the Panthalassic Ocean during the latest Permian: Geology, v. 38, p. 187-190.

Algeo, T.J., Kuwahara, K., Sano, H., Bates, S., Lyons, T., Elswick, E., Hinnov, L., Ellwood, B.B., Moser, J., and Maynard, J.B., 2010b, Spatial variation in sediment fluxes, redox conditions, and productivity in the Permian-Triassic Panthalassic Ocean: Palaeogeography, Palaeoclimatology, Palaeoecology, in press.

Algeo, T.J., Shen, Y., Zhang, T., Lyons, T.W., Bates, S.M., Rowe, H., and Nguyen, T.K.T., 2008, Association of ${ }^{34}$ S-depleted pyrite layers with negative carbonate $\delta^{13} \mathrm{C}$ excursions at the Permian/Triassic boundary: Evidence for upwelling of sulfidic deep-ocean watermasses: Geochemistry Geophysics Geosystems, v. 9, 10 pp., Q04025, doi:10.1029/2007GC001823.

Cassinis, G., and Perotti, C.R., 2007, A stratigraphic and tectonic review of the Italian Southern Alpine Permian: Palaeoworld, v. 16, p. $140-172$.

Chen, J., Beatty, T.W., Henderson, C.M., and Rowe, H., 2009, Conodont biostratigraphy across the Permian-Triassic boundary at the Dawen section, Great Bank of Guizhou, Guizhou Province, South China: Implications for the Late Permian extinction and correlation with Meishan: Journal of Asian Earth Sciences, v. 36, p. 442-458.

Farabegoli, E., Perri, M.C., and Posenato, R., 2007, Environmental and biotic changes across the Permian-Triassic boundary in western Tethys: The Bulla parastratotype, Italy: Global and Planetary Change, v. 55, p. 109-135.

Grasby, S.E., and Beauchamp, B., 2009, Latest Permian to Early Triassic basin-to-shelf anoxia in the Sverdrup Basin, Arctic Canada: Chemical Geology, v. 264, p. 232-246.

Hays, L.E., Beatty, T., Henderson, C.M., Love, G.D., and Summons, R.E., 2007, Evidence for photic zone euxinia through the endPermian mass extinction in the Panthalassic Ocean (Peace River Basin, Western Canada): Paleaoworld, v. 16, p. 39-50.

Henderson, C.M., 1997, Uppermost Permian conodonts and the Permian-Triassic boundary in the Western Canada Sedimentary Basin: Bulletin of Canadian Petroleum Geology, v. 45, p. 693-707.

Henderson, C.M., and Baud, A., 1997, Correlation of the Permian-Triassic boundary in Arctic Canada and comparison with Meishan, China. In: Wang, N., and Remane, J. (Eds.), Proceedings of the $30^{\text {th }}$ International Geological Congress, v. 11, pp. $143-152$. 
Horacek, M., Brandner, R., and Abart, R., 2007, Carbon isotope record of the P/T boundary and the Lower Triassic in the Southern Alps: Evidence for rapid changes in storage of organic carbon: Palaeogeography, Palaeoclimatology, Palaeoecology, v. 252, p. 347354.

Horacek, M., Richoz, S., Brandner, R., Krystyn, L., and Spötl, C., 2007b, Evidence for recurrent changes in Lower Triassic oceanic circulation of the Tethys: The $\delta^{13} \mathrm{C}$ record from marine sections in Iran: Palaeogeography, Palaeoclimatology, Palaeoecology, v. 252, p. 355-369.

Korte, C., Kozur, H.W., and Partoazar, H., 2004, Negative carbon isotope excursion at the Permian/Triassic boundary section at Zal, NW-Iran: Hallesches Jahrbuch der Geowissenschaften, v. B18, p. 69-71.

Korte, C., Pande, P., Kalia, P., Kozur, H.W., Joachimski, M.M., and Oberhänsli, H., 2010, Massive volcanism at the Permian-Triassic boundary and its impact on the isotopic composition of the ocean and atmosphere: Journal of Asian Earth Sciences, v. 37, p. 293311.

Kozur, H.W., 2007, Biostratigraphy and event stratigraphy in Iran around the Permian-Triassic boundary (PTB): Implications for the causes of the PTB biotic crisis: Global and Planetary Change, v. 55, p. 155-176.

Krystyn, L., Bhargava, O.N., and Richoz, S., 2007, A candidate GSSP for the base of the Olenekian Stage: Mud at Pin Valley; district Lahul \& Spiti, Himachal Pradesh (Western Himalaya), India: Albertiana, v. 35, p. 5-29.

Lehrmann, D.J., Wan, Y., Wei, J., Yu, Y.Y., and Xiao, J., 2001, Lower Triassic peritidal cyclic limestone: an example of anachronistic carbonate facies from the Great Bank of Guizhou, Nanpanjiang Basin, Guizhou province, South China: Palaeogeography, Palaeoclimatology, Palaeoecology, v. 173, p. 103-123.

Li, S., Tong, J., Liu, K., Wang, F., and Huo, Y., 2007, The Lower Triassic cyclic deposition in Chaohu, Anhui Province, China: Palaeogeography, Palaeoclimatology, Palaeoecology, v. 252, p. 188-199.

Luu, T.P.L., Ellwood, B.B., Tomkin, J.H., and Truong, D.N., 2008, A magnetic susceptibility study of the Permian/Triassic boundary limestone section at Ha Giang, Vietnam and graphic comparison to the Meishan, China GSSP: Palaeogeography, Palaeoclimatology, Palaeoecology, in review.

Orchard, M.J., and Krystyn, L., 1998, Conodonts of the lowermost Triassic of Spiti, and new zonation based on Neogondolella successions: Rivista italiana di paleontologia e stratifigrafia, v. 104, p. 341-368.

Son, T.H., Koeberl, C., Ngoc, N.L., and Huyen, D.T., 2007, The Permian-Triassic boundary sections in northern Vietnam (Nhi Tao and Lung Cam sections): Carbon-isotope excursion and elemental variations indicate major anoxic event: Palaeoworld, v. 16, p. 5166.

Twitchett, R.J., and Wignall, P.B., 1996, Trace fossils and the aftermath of the Permo-Triassic mass extinction: Evidence from northern Italy: Palaeogeography, Palaeoclimatology, Palaeoecology, v. 124, p. 137-151.

Wignall, P.B., and Newton, R., 2003, Contrasting deep-water records from the Upper Permian and Lower Triassic of South Tibet and British Columbia: Evidence for a diachronous mass extinction: Palaios, v. 18, p. 153-167. 
Zhao, L., Orchard, M.J., Tong, J., Sun, Z., Zuo, J., Zhang, S., and Yun, A., 2007, Lower Triassic conodont sequence in Chaohu, Anhui

Province, China and its global correlation: Palaeogeography, Palaeoclimatology, Palaeoecology, v. 252, p. 24-38.

Table DR2. Sediment fluxes ${ }^{1}$ for Changhsingian (uppermost Permian) and Griesbachian (lowermost Triassic) units

\begin{tabular}{|c|c|c|c|c|c|c|c|c|c|c|c|c|c|}
\hline \multirow[b]{2}{*}{ No } & \multirow[b]{2}{*}{ Section } & \multicolumn{4}{|c|}{ CHANGXINGIAN } & \multicolumn{4}{|c|}{ GRIESBACHIAN } & \multicolumn{4}{|c|}{$\mathrm{Gr} / \mathrm{Ch}^{2}$} \\
\hline & & $\begin{array}{l}\text { BAR } \\
\mathrm{g} \mathrm{m}^{-2} \\
\mathrm{yr}^{-1}\end{array}$ & $\begin{array}{l}\mathrm{CaCO}_{3} \\
\mathrm{~g} \mathrm{~m}^{-2} \mathrm{yr}^{-1}\end{array}$ & $\begin{array}{l}\text { Quartz } \\
\mathrm{g} \mathrm{m}^{-2} \mathrm{yr}^{-1}\end{array}$ & $\begin{array}{l}\text { Clays } \\
\mathrm{g} \mathrm{m}_{1}^{-2} \mathrm{yr}^{-}\end{array}$ & $\begin{array}{c}\text { BAR } \\
\mathrm{g} \mathrm{m}_{1}^{-2} \mathrm{yr}^{-}\end{array}$ & $\begin{array}{l}\mathrm{CaCO}_{3} \\
\mathrm{~g} \mathrm{~m}_{1}^{-2} \mathrm{yr}^{-}\end{array}$ & $\begin{array}{l}\text { Quart } \\
\mathbf{z} \\
\mathrm{g} \mathrm{m}_{1}^{-2} \mathrm{yr}^{-}\end{array}$ & $\begin{array}{c}\text { Clays } \\
\mathrm{g} \mathrm{m}^{-2} \\
\mathrm{yr}^{-1}\end{array}$ & $\begin{array}{c}\text { BAR } \\
\%\end{array}$ & $\begin{array}{c}\mathrm{CaC} \\
\mathbf{O}_{3} \\
\%\end{array}$ & $\begin{array}{c}\text { Quart } \\
\text { Z } \\
\%\end{array}$ & $\begin{array}{c}\text { Clay } \\
\mathbf{S} \\
\%\end{array}$ \\
\hline 1 & Chaohu, Anhui & 35.3 & 10.21 & 13.67 & 11.42 & 214.3 & 54.39 & 36.70 & 123.19 & 507 & 433 & 168 & 979 \\
\hline 2 & Dawen, Guizhou & 88.2 & 85.51 & 0.76 & 1.97 & 571.4 & 545.91 & 0.87 & 24.65 & 548 & 538 & 15 & 1150 \\
\hline 3 & Nhi Tao, Vietnam & 107.3 & 99.11 & 1.78 & 6.35 & 240.0 & 212.16 & 3.32 & 24.52 & 124 & 114 & 86 & 286 \\
\hline 4 & Lung Cam, Vietnam & 107.3 & 95.19 & 7.44 & 4.62 & 240.0 & 211.02 & 5.13 & 23.85 & 124 & 122 & -31 & 416 \\
\hline 5 & Spiti, India & 0.7 & 0.06 & 0.14 & 0.53 & 3.6 & 2.51 & 0.28 & 0.79 & 386 & 4176 & 93 & 47 \\
\hline 6 & Guryul Ravine, India & 14.7 & 5.02 & 5.23 & 4.46 & 28.6 & 4.97 & 5.84 & 17.77 & 94 & -1 & 12 & 299 \\
\hline 7 & Zal, Iran & 29.4 & 27.93 & $n / a^{3}$ & 1.48 & 428.6 & 398.27 & $\mathrm{n} / \mathrm{a}^{3}$ & 30.30 & 1357 & 1326 & $n / a^{3}$ & 1944 \\
\hline 8 & Bulla, Italy & 88.2 & 83.11 & $\mathrm{n} / \mathrm{a}^{3}$ & 5.13 & 321.4 & 270.34 & $\mathrm{n} / \mathrm{a}^{3}$ & 51.08 & 264 & 225 & $\mathrm{n} / \mathrm{a}^{3}$ & 897 \\
\hline 9 & Uomo, Italy & 88.2 & 86.92 & $\mathrm{n} / \mathrm{a}^{3}$ & 1.31 & 321.4 & 277.61 & $\mathrm{n} / \mathrm{a}^{3}$ & 43.82 & 264 & 219 & $n / a^{3}$ & 3244 \\
\hline 10 & Spitsbergen & 29.4 & 3.81 & 15.29 & 10.31 & 214.3 & 52.37 & 84.86 & 77.06 & 629 & 1273 & 455 & 648 \\
\hline 11 & $\begin{array}{l}\text { West Blind Fiord, } \\
\text { Arctic }\end{array}$ & 14.7 & 0.10 & 6.12 & 8.49 & 357.1 & 9.13 & 73.96 & 274.06 & 2329 & 9279 & 1108 & 3129 \\
\hline 12 & Ursula Creek & 5.0 & 0.57 & 3.88 & 0.55 & 357.1 & 58.42 & 155.16 & 143.56 & 7043 & 10064 & 3900 & $\begin{array}{r}2620 \\
1\end{array}$ \\
\hline 13 & $\begin{array}{l}\text { Crooked Creek, } \\
\text { Alberta }\end{array}$ & 5.0 & 1.79 & 2.15 & 1.06 & 285.7 & 67.82 & 118.96 & 98.93 & 5614 & 3679 & 5438 & 9258 \\
\hline 14 & Opal Creek, Alberta & 5.0 & 1.56 & 1.34 & 2.10 & 285.7 & 53.49 & 78.81 & 153.41 & 5614 & 3336 & 5772 & 7203 \\
\hline 15 & $\begin{array}{l}\text { Gujo-Hachiman, } \\
\text { Japan }\end{array}$ & 15.9 & 0.00 & 14.44 & 1.47 & 4.1 & 0.00 & 2.19 & 1.87 & -74 & 0 & -85 & 27 \\
\hline 16 & Ubara, Japan & 11.3 & 0.00 & 9.38 & 1.87 & 5.8 & 0.00 & 3.55 & 2.28 & -48 & 0 & -62 & 22 \\
\hline
\end{tabular}


${ }^{1}$ Bulk accumulation rates (BAR) calculated per equation 2 and component fluxes calculated per equation 3 of Methods Supplement.

${ }^{2}$ Represents the percentage changes in BAR and sediment component fluxes from the Changxingian (Ch) to the $\mathrm{Griesbachian}(\mathrm{Gr})$.

${ }^{3}$ Clay fluxes represent all siliciclastic components (i.e., quartz and clays not separately determined).

Table DR3. Lithologic compositions ${ }^{1}$ of Changhsingian (uppermost Permian) and Griesbachian (lowermost Triassic) units

\begin{tabular}{|c|c|c|c|c|c|c|c|c|c|}
\hline \multirow[b]{2}{*}{ No } & \multirow[b]{2}{*}{ Section } & \multicolumn{4}{|c|}{ CHANGXINGIAN } & \multicolumn{4}{|c|}{ GRIESBACHIAN } \\
\hline & & $\mathbf{n}$ & $\begin{array}{c}\mathrm{CaCO}_{3} \\
\%\end{array}$ & $\begin{array}{c}\text { Quartz } \\
\%\end{array}$ & $\begin{array}{c}\text { Clays } \\
\%\end{array}$ & $\mathbf{n}$ & $\begin{array}{c}\mathrm{CaCO}_{3} \\
\%\end{array}$ & $\begin{array}{c}\text { Quartz } \\
\%\end{array}$ & $\begin{array}{c}\text { Clays } \\
\%\end{array}$ \\
\hline 1 & Chaohu, Anhui & 6 & $28.6( \pm 37.3)$ & $38.3( \pm 25.2)$ & $31.9( \pm 18.6)$ & 36 & $25.5( \pm 22.2)$ & $17.2( \pm 9.4)$ & $57.8( \pm 18.9)$ \\
\hline 2 & Dawen, Guizhou & $\begin{array}{l}2 \\
6 \\
\end{array}$ & $96.9( \pm 3.8)$ & $0.9( \pm 4.0)$ & $2.2( \pm 1.5)$ & 49 & $95.5( \pm 2.0)$ & $0.2( \pm 0.3)$ & $4.3( \pm 2.1)$ \\
\hline 3 & Nhi Tao, Vietnam & $\begin{array}{l}2 \\
7 \\
\end{array}$ & $92.0( \pm 1.8)$ & $1.6( \pm 1.9)$ & $5.9( \pm 2.1)$ & 69 & $88.2( \pm 3.0)$ & $1.4( \pm 1.5)$ & $10.2( \pm 3.1)$ \\
\hline 4 & Lung Cam, Vietnam & $\begin{array}{l}5 \\
5 \\
\end{array}$ & $88.6( \pm 21.2)$ & $6.9( \pm 18.1)$ & $4.3( \pm 10.0)$ & 46 & $87.5( \pm 5.1)$ & $2.1( \pm 1.4)$ & $9.9( \pm 3.8)$ \\
\hline 5 & Spiti, India & $\begin{array}{l}2 \\
2 \\
\end{array}$ & $7.9( \pm 24.5)$ & $19.2( \pm 8.0)$ & $71.6( \pm 20.6)$ & 6 & $70.0( \pm 35.4)$ & $7.7( \pm 9.3)$ & $21.9( \pm 33.6)$ \\
\hline 6 & Guryul Ravine, India & 8 & $34.1( \pm 32.0)$ & $35.6( \pm 11.8)$ & $30.3( \pm 33.2)$ & 11 & $17.4( \pm 29.0)$ & $20.4( \pm 5.2)$ & $62.2( \pm 32.2)$ \\
\hline 7 & Zal, Iran & 3 & $94.8( \pm 9.6)$ & $\mathrm{n} / \mathrm{a}^{2}$ & $5.0( \pm 9.5)^{2}$ & 92 & $92.8( \pm 9.6)$ & $\mathrm{n} / \mathrm{a}^{2}$ & $7.1( \pm 9.5)^{2}$ \\
\hline 8 & Bulla, Italy & $\begin{array}{l}3 \\
9\end{array}$ & $93.9( \pm 6.3)$ & $\mathrm{n} / \mathrm{a}^{2}$ & $5.8( \pm 6.4)^{2}$ & 11 & $83.8( \pm 12.5)$ & $\mathrm{n} / \mathrm{a}^{2}$ & $15.8( \pm 12.4)^{2}$ \\
\hline 9 & Uomo, Italy & $\begin{array}{l}1 \\
4 \\
\end{array}$ & $98.2( \pm 1.7)$ & $\mathrm{n} / \mathrm{a}^{2}$ & $1.5( \pm 1.6)^{2}$ & 5 & $86.2( \pm 12.8)$ & $\mathrm{n} / \mathrm{a}^{2}$ & $13.6( \pm 12.8)^{2}$ \\
\hline 10 & Spitsbergen & $\begin{array}{l}2 \\
8 \\
\end{array}$ & $12.8( \pm 24.3)$ & $51.4( \pm 22.8)$ & $34.6( \pm 22.6)$ & 60 & $23.7( \pm 21.2)$ & $38.4( \pm 15.3)$ & $34.8( \pm 15.7)$ \\
\hline 11 & $\begin{array}{l}\text { West Blind Fiord, } \\
\text { Arctic }\end{array}$ & $\begin{array}{l}3 \\
6 \\
\end{array}$ & $0.7( \pm 1.4)$ & $41.6( \pm 29.0)$ & $57.7( \pm 29.3)$ & 20 & $2.6( \pm 2.0)$ & $20.7( \pm 5.8)$ & $76.7( \pm 7.7)$ \\
\hline 12 & Ursula Creek & $\begin{array}{l}1 \\
2 \\
\end{array}$ & $11.3( \pm 27.9)$ & $76.6( \pm 27.0)$ & $10.8( \pm 9.9)$ & 29 & $16.0( \pm 25.7)$ & $42.6( \pm 21.8)$ & $39.4( \pm 21.2)$ \\
\hline 13 & Crooked Creek, & 3 & $33.0( \pm 22.5)$ & $39.5( \pm 12.4)$ & $19.4( \pm 16.5)$ & 60 & $22.9( \pm 5.6)$ & $40.2( \pm 6.6)$ & $33.4( \pm 8.0)$ \\
\hline
\end{tabular}




\begin{tabular}{|c|l|r|c|c|c|c|c|c|c|}
\hline & Alberta & & & & & & & & \\
\hline 14 & Opal Creek, Alberta & 4 & $30.1( \pm 5.8)$ & $25.9( \pm 1.0)$ & $40.6( \pm 6.1)$ & 92 & $17.9( \pm 9.3)$ & $26.4( \pm 6.2)$ & $51.4( \pm 8.0)$ \\
\hline \multirow{2}{*}{15} & Gujo-Hachiman, & 8 & & & & & 13 & & \\
& Japan & 8 & $0( \pm 0)$ & $89.4( \pm 5.4)$ & $9.1( \pm 5.2)$ & & $0( \pm 0)$ & $51.1( \pm 8.0)$ & $43.5( \pm 7.5)$ \\
\hline \multirow{2}{*}{16} & Ubara, Japan & 9 & $0( \pm 0)$ & $81.1( \pm 5.6)$ & $16.1( \pm 6.4)$ & 6 & $0( \pm 0)$ & $57.8( \pm 4.3)$ & $37.2( \pm 6.8)$ \\
\hline
\end{tabular}

${ }^{1}$ Calculated per Equations 4-6 in Methods Section.

${ }^{2}$ Clay percentages represent all siliciclastic components (i.e., quartz and clays not separately determined). 
Table DR4. Biological effects of sedimentation

\begin{tabular}{|c|c|c|c|c|}
\hline Organism & Locale & Metric & Comments & Data Source \\
\hline $\begin{array}{l}\text { Scleractinian } \\
\text { corals }\end{array}$ & $\begin{array}{l}\text { experiment } \\
\text { al }\end{array}$ & growth rate & $\begin{array}{l}\text { Application of } 200 \mathrm{mg} \mathrm{cm}-2 \text { of sandy sediment to Astrangia daily for four weeks, then thrice daily for four } \\
\text { weeks (final two weeks without food). Growth rates slowed in response to thrice-daily sediment } \\
\text { treatments and again in response to food limitation; all colonies recovered after 8-week stress period } \\
\text { terminated. }\end{array}$ & Peters \& Pilson (1985) \\
\hline $\begin{array}{l}\text { Scleractinian } \\
\text { corals }\end{array}$ & metastudy & growth rate & $\begin{array}{l}\text { Documentation of adverse effects of "heavy" sedimentation }\left(>10 \mathrm{mg} \mathrm{cm}^{-2}\right) \text { and suspended sediment } \\
\text { concentrations }\left(>10 \mathrm{mg} \mathrm{L}^{-1}\right) \text {, including decreased productivity and calcification, increased respiration, } \\
\text { and mortality. Primary studies documented stress in various coral species at sediment fluxes mainly } \\
\text { from } 100 \text { to } 1000 \mathrm{mg} \mathrm{cm}^{-2} \mathrm{~d}^{-1} \text {. }\end{array}$ & Rogers (1990) \\
\hline $\begin{array}{l}\text { Scleractinian \& } \\
\text { alcyonacean } \\
\text { corals }\end{array}$ & $\begin{array}{l}\text { experiment } \\
\text { al }\end{array}$ & growth rate & $\begin{array}{l}\text { Application of } 100 \text { to } 400 \mathrm{mg} \mathrm{cm}-2 \text { pulse of sandy sediment resulted in a } 75 \% \text { to } 97 \% \text { reduction in light } \\
\text { reception by coral zooxanthellae. A sediment application of } 200 \mathrm{mg} \mathrm{cm}-2 \text { produced a } \sim 3 \text { to } 6 \mathrm{X} \\
\text { decrease in growth rates at low light levels and a } \sim 1.5 \text { to } 2.0 \mathrm{X} \text { decrease at high light levels. }\end{array}$ & Riegl \& Branch (1995) \\
\hline $\begin{array}{l}\text { Scleractinian } \\
\text { corals, barnacles } \\
\quad \& \text { copepods }\end{array}$ & $\begin{array}{l}\text { Great } \\
\text { Barrier Reef }\end{array}$ & $\begin{array}{l}\text { self- } \\
\text { cleaning } \\
\text { potential }\end{array}$ & $\begin{array}{l}\text { Application of muddy sediment to investigate "self-cleaning" capacity: at }<0.5 \mathrm{mg} \mathrm{cm}^{-2} \text {, organisms self- } \\
\text { cleaned effectively; at } 4-5 \mathrm{mg} \mathrm{cm} \mathrm{cm}^{-2} \text {, organisms exhibited stressed behaviors, and barnacles and } \\
\text { calanoid copepods died after }<1 \mathrm{hr} \text {. Stresses more severe when mud flocculated to form "marine } \\
\text { snow." }\end{array}$ & Fabricius \& Wolanski (2000) \\
\hline $\begin{array}{l}\text { Scleractinian } \\
\text { corals }\end{array}$ & $\begin{array}{l}\text { experiment } \\
\text { al, Great } \\
\text { Barrier Reef }\end{array}$ & growth rate & $\begin{array}{l}\text { Application of } \sim 100-200 \mathrm{mg} \mathrm{cm}^{-2} \text { of muddy sediment caused a significant reduction in growth rate and } \\
\text { local mortality in Porites, Montipora, and other corals. Harmful effects increased directly with sediment } \\
\text { load and length of exposure and inversely with grain size and were positively correlated with cumulative } \\
\text { "exposure" (i.e., hours x sediment flux). }\end{array}$ & Philipp \& Fabricius (2003) \\
\hline $\begin{array}{l}\text { Scleractinian } \\
\text { corals }\end{array}$ & metastudy & $\begin{array}{l}\text { growth rate, } \\
\text { reproductio } \\
\quad \mathrm{n} \& \\
\text { recruitment }\end{array}$ & $\begin{array}{l}\text { Documentation of patterns of physiological response of coral colonies and other benthic marine } \\
\text { organisms to changes in sediment flux, light levels, nutrient levels, boring and predation, and other } \\
\text { factors in both natural and artificial settings. Significant stresses in coral colonies and reduced larval } \\
\text { recruitment at sediment fluxes }>10 \mathrm{~g} \mathrm{~cm}^{-2} \mathrm{~d}^{-1} \text {. }\end{array}$ & Fabricius (2005) \\
\hline $\begin{array}{l}\text { Scleractinian } \\
\text { corals }\end{array}$ & $\begin{array}{l}\text { experiment } \\
\quad \text { al }\end{array}$ & $\begin{array}{l}\text { growth rate, } \\
\text { self- } \\
\text { cleaning } \\
\text { potential }\end{array}$ & $\begin{array}{l}\text { Application of } 33-160 \mathrm{mg} \mathrm{cm}^{-2} \text { of various sediment types with varying nutrient levels demonstrated (1) } \\
\text { extended exposure ( }>12 \mathrm{~h} \text { ) resulted in large increases in stress; (2) stress was directly proportional to } \\
\text { cumulative "exposure" (i.e., hours } x \text { sediment flux); (3) stress increased at finer grain sizes owing to } \\
\text { onset of anoxia in the sediment layer. }\end{array}$ & Weber et al. (2006) \\
\hline $\begin{array}{l}\text { Cyanobacteria } \\
\text { (Synechococcus) }\end{array}$ & $\begin{array}{l}\text { South } \\
\text { China Sea }\end{array}$ & growth rate & $\begin{array}{l}\text { Varied from } \sim 1.2 \mathrm{~d}^{-1} \text { to } \sim 0.2 \mathrm{~d}^{-1} \text { at concentrations of total suspended solids (TSS) ranging from }<0.5 \mathrm{mg} \\
\mathrm{L}^{-1} \text { to } 2.0 \mathrm{mg} \mathrm{L} \\
\mathrm{I}^{-1} \text {; suspended solids reduced ambient light levels and photosynthetic rates. }\end{array}$ & Agawin et al. (2003) \\
\hline $\begin{array}{l}\text { Benthic } \\
\text { foraminifera }\end{array}$ & $\begin{array}{l}\text { South } \\
\text { China Sea }\end{array}$ & abundance & $\begin{array}{l}\text { Complete mortality of benthic foraminifera in proximal areas receiving } 8-10 \mathrm{~cm} \text { of ash from the } 1991 \mathrm{Mt} \text {. } \\
\text { Pinatubo eruption, and marked declines in abundance and diversity in distal areas receiving } 1-2 \mathrm{~cm} \text { of } \\
\text { ash. }\end{array}$ & $\begin{array}{l}\text { Marquez (2000); Kuhnt et al. } \\
\text { (2005) }\end{array}$ \\
\hline Freshwater algae & $\begin{array}{l}\text { experiment } \\
\quad \text { al }\end{array}$ & growth rate & $\begin{array}{l}\text { Varied by a factor of } \sim 12 \text { as a consequence of clay deposition rates from } 30 \text { to } 250 \mathrm{~g} \mathrm{~m}^{-2} \text { during a 3-day } \\
\text { stress period. High sedimentation rates also caused a 3-4X increase in the abundance of cyanobacteria } \\
\text { relative to green algae. }\end{array}$ & Izagirre et al. (2009) \\
\hline
\end{tabular}




\begin{tabular}{|c|c|c|c|c|}
\hline $\begin{array}{l}\text { Freshwater } \\
\text { mussels }\end{array}$ & Wisconsin & abundance & $\begin{array}{l}\text { TSS increased from } \sim 0.01-0.1 \mathrm{~g} \mathrm{~L}^{-1} \text { to } \sim 0.1-1 \mathrm{~g} \mathrm{~L}^{-1} \text { at a site } 0.5 \mathrm{~km} \text { downstream of a removed dam, } \\
\text { concurrent with a decrease in mussel abundance from } 3.8 \mathrm{~m}^{-2} \text { to } 2.6 \mathrm{~m}^{-2} \text { but only limited changes in } \\
\text { diversity (loss of a single species). }\end{array}$ & Sethi et al. (2004) \\
\hline Shrimps & $\begin{array}{l}\text { experiment } \\
\text { al }\end{array}$ & $\begin{array}{l}\text { osmo- } \\
\text { regulation } \\
\text { and survival }\end{array}$ & $\begin{array}{l}\text { At sediment concentrations above } 0.9 \mathrm{gL}-1 \text { ( } 65 \pm 15 \mathrm{NTU} \text { ), juveniles of the shrimp Penaeus japonicus } \\
\text { suffered significant increases in mortality and a decreased tolerance to salinity fluctuations. Feeding } \\
\text { activity was reduced in concentrations above } 0.15 \mathrm{gL}-1 \text { ( } 35 \pm 15 \mathrm{NTU}) \text {. }\end{array}$ & Lin et al. (1992) \\
\hline Scallops & $\begin{array}{c}\text { Southern } \\
\text { Brazil }\end{array}$ & growth rate & $\begin{array}{l}\text { Sea-based nursery experiments on cultures of the lion's paw scallop Nodipecten nodosus demonstrated } \\
\text { a statistically significant negative relationship between turbidity and daily growth rate. }\end{array}$ & Rupp et al. (2005) \\
\hline $\begin{array}{l}\text { Benthic } \\
\text { ecosystems }\end{array}$ & Hawaii & $\begin{array}{l}\text { ecosystem } \\
\text { structure } \\
\text { and } \\
\text { function }\end{array}$ & $\begin{array}{l}\text { Field surveys in Kaneohe Bay, Hawaii, recorded a low diversity, small-sized, low biomass, low } \\
\text { productivity macrobenthic community dominated by very small, deposit-feeding polychaetes. This } \\
\text { unusual community structure was attributed to the high rate of sedimentation in the study site (locally } \\
\text { upto } 6-7 \mathrm{~cm} \mathrm{yr}^{-1} \text { ). }\end{array}$ & Smith and Kukert (1996) \\
\hline Amphibians & $\begin{array}{l}\text { New South } \\
\text { Wales }\end{array}$ & $\begin{array}{l}\text { growth and } \\
\text { developme } \\
\text { nt }\end{array}$ & $\begin{array}{l}\text { Addition of a } 1.25 \mathrm{~mm} \text { deep layer of silt to stream enclosures containing tadpoles of the frog Litoria } \\
\text { spenceri caused a significant reduction in body length and development. Two subsequent additions of } \\
\text { silt at 3-weekly intervals caused further reductions in growth and development }\end{array}$ & Gillespie (2002) \\
\hline $\begin{array}{l}\text { Freshwater } \\
\text { gastropods }\end{array}$ & $\begin{array}{l}\text { Lake } \\
\text { Tanganyika } \\
\& \\
\text { experiment } \\
\quad \text { al }\end{array}$ & $\begin{array}{l}\text { size and } \\
\text { survivorship }\end{array}$ & $\begin{array}{l}\text { Additions of } 80 \mathrm{~kg} \text { sand } \mathrm{m}^{-2} \text { (a } 2-3 \mathrm{~cm} \text { deep layer) to rocky shore ecosystems resulted in a significant } \\
\text { reduction in the body size of Lavigeria spp. Laboratory experiments showed that all size classes of } \\
\text { Lavigeria grandis had significantly lower survivorship in sediment-impacted treatments, with larger } \\
\text { individuals being affected more than smaller ones. }\end{array}$ & Donohue and Irvine (2004) \\
\hline Infaunal clams & $\begin{array}{l}\text { experiment } \\
\text { al }\end{array}$ & growth rate & $\begin{array}{l}\text { Experiments using mixed algae-silt suspensions demonstrated that growth rates in juvenile Mercenaria } \\
\text { mercenaria declined significantly when silt concentration exceeded } 0.044 \mathrm{gL}^{-1} \text {. Experimental growth } \\
\text { rates were comparable to wild populations in Great South Bay, New York under similar conditions. }\end{array}$ & Bricelj et al. (1984) \\
\hline Infaunal clams & $\begin{array}{l}\text { experiment } \\
\text { al }\end{array}$ & feeding & $\begin{array}{l}\text { Experiments under increasing particulate concentrations showed a significant reduction in energy intake } \\
\text { by Cerastoderma edule at concentrations above } 0.3 \mathrm{gL}^{-1} \text {, due to reductions in filtration rate, ingestion } \\
\text { rate and absorption efficiency }\end{array}$ & $\begin{array}{l}\text { Navarro and Widdows } \\
(1997)\end{array}$ \\
\hline Oysters & $\begin{array}{l}\text { experiment } \\
\text { al }\end{array}$ & feeding & $\begin{array}{l}\text { Application of increasing concentrations of silt, kaolin and calcium carbonate caused a reduction in the } \\
\text { rate of filter feeding (the rate that water is pumped across the gills) in Ostrea virginica. Pumping rate } \\
\text { decreased by }>80 \% \text { under turbidity of } 1 \mathrm{gL}^{-1} \text { silt, and }>90 \% \text { under } 3 \mathrm{gL}^{-1} \text { silt. }\end{array}$ & $\begin{array}{l}\text { Loosanoff and Tommers } \\
\text { (1948) }\end{array}$ \\
\hline
\end{tabular}




\section{References for Table DR4)}

Agawin, N.S.R., Duarte, C.M., Agustí, S., and McManus, L., 2003, Abundance, biomass and growth rates of Synechococcus sp. in a tropical coastal ecosystem (Philippines, South China Sea): Estuarine Coastal and Shelf Science, v. 56, p. 493-502.

Bricelj, V.M., Malouf, R.E., and de Quillfeldt, C., 1984, Growth of juvenile Mercenaria mercenaria and the effect of resuspended bottom sediments: Marine Biology, v. 84, p. 167-173.

Donohue, I., and Irvine, K., 2004, Size-specific effects of increased sediment loads on gastropod communities in Lake Tanganyika, Africa: Hydrobiologia, v. 522, p. 337-342.

Fabricius, K.E., 2005, Effects of terrestrial runoff on the ecology of corals and coral reefs: review and synthesis: Marine Pollution Bulletin, v. 50, p. 125-146.

Fabricius, K.E., and Wolanski, E., 2000, Rapid smothering of coral reef organisms by muddy marine snow: Estuarine Coastal and Shelf Science, v. 50, p. 115-120.

Gillespie, G.R., 2002, Impacts of sediment loads, tadpole density, and food type on the growth and development of tadpoles of the spotted tree frog Litoria spenceri: an in-stream experiment: Biological Conservation, v. 106, p. 141-150.

Izagirre, O., Serra, A., Guasch, H., and Elosegi, A., 2009, Effects of sediment deposition on periphytic biomass, photosynthetic activity and algal community structure: Science of the Total Environment, v. 407, p. 5694-5700.

Kuhnt, W., Hess, S., Hobourn, A., Paulsen, H., and Salomon, B., 2005, The impact of the 1991 Mt. Pinatubo eruption on deep-sea foraminiferal communities: A model for the Cretaceous-Tertiary $(\mathrm{K} / \mathrm{T})$ boundary?: Palaeogeography, Palaeoclimatology, Palaeoecology, v. 224, p. 83-107.

Lin , H.-P., Charmantier, G., Thuet, P., and Trilles, J.-P., 1992, Effects of turbidity on survival osmoregulation and gill Na ${ }^{+}-\mathrm{K}^{+}$ ATPase in juvenile shrimp Penaeus japonicus: Marine Ecology Progress Series, v. 90, p. 31-37.

Loosanoff, V.L., and Tommers, F.D., 1948, Effect of suspended silt and other substances on rate of feeding of oysters: Science, v. 107, p. 69-70.

Marquez, E.J., 2000, The 1991 Mount Pinatubo eruption and eastern South China Sea foraminifera: occurrence, composition and recovery: The Island Arc, v. 9, p. 527-541.

Navarro, J.M., and Widdows, J., 1997, Feeding physiology of Cerastoderma edule in response to a wide range of seston concentrations: Marine Ecology Progress Series, v. 152, p. 175-186

Peters, E.C., and Pilson, M.E.Q., 1985, A comparative study of the effects of sedimentation on symbiotic and asymbiotic colonies of the coral Astrangia danae Milne Edwards and Haime 1849: Journal of Experimental Marine Biology and Ecology, v. 92, p. 215230 .

Philipp, E., and Fabricius, K., 2003, Photophysiological stress in scleractinian corals in response to short-term sedimentation: Journal of Experimental Marine Biology and Ecology, v. 287, p. 57-78. 
Riegl, B., and Branch, G.M., 1995, Effects of sediment on the energy budgets of four scleractinian (Bourne 1900) and five alcyonacean (Lamouroux 1816) corals: Journal of Experimental Marine Biology and Ecology, v. 186, p. 259-275.

Rogers, C.S., 1990, Responses of coral reefs and reef organisms to sedimentation: Marine Ecology Progress Series, v. 62, p. 185-202.

Rupp, G.S., Parsons, G.J., Thompson, R.J., and de Bem, M.M., 2005, Effect of depth and stocking density on growth and retrieval of the postlarval lion's paw scallop, Nodipecten nodosus (Linnaeus, 1758): Journal of Shellfish Research, v. 23, p. 473-482.

Sethi, S.A., Selle, A.R., Doyle, M.W., Stanley, E.H., and Kitchel, H.E., 2004, Response of unionid mussels to dam removal in Koshkonong Creek, Wisconsin (USA): Hydrobiologia, v. 525, p. 157-165.

Smith, C.R., and Kukert, H., 1996, Macrobenthic community structure, secondary production, and rates of bioturbation and sedimentation at the Kāne'ohe Bay lagoon floor: Pacific Science, v. 50, p. 211-219.

Weber, M., Lott, C., and Fabricius, K.E., 2006, Sedimentation stress in a scleractinian coral exposed to terrestrial and marine sediments with contrasting physical, organic and geochemical properties: Journal of Experimental Marine Biology a 